Research Article

\title{
Perceived Psychosocial Benefits for Individuals with Parkinson's Disease Following Therapeutic Yoga: A Qualitative Analysis
}

\author{
Em V. Adams ${ }^{1,}{ }^{*}$, Marieke Van Puymbroeck ${ }^{2}$, Brent L. Hawkins ${ }^{3}$, Arlene A. Schmid ${ }^{4}$
}

1. Herbert H. Lehman College, Bronx, New York, USA; E-Mail: emilie.adams@lehman.cuny.edu

2. Clemson University, Clemson, SC, USA; E-Mail: mvp@clemson.edu

3. University of North Carolina, Wilmington, USA; E-Mail: hawkinsb@uncw.edu

4. Colorado State University, Fort Collins, CO, USA; E-Mail: Arlene.schmid@colostate.edu

* Correspondence: Em V. Adams; E-Mails: emilie.adams@lehman.cuny.edu; emiliea@clemson.edu

Academic Editor: Marieke Van Puymbroeck

Special Issue: Yoga in Older Adults

OBM Geriatrics

2020 , volume 4 , issue 4

doi:10.21926/obm.geriatr.2004144
Received: August 19, 2020

Accepted: December 14, 2020

Published: December 22, 2020

\begin{abstract}
Using a qualitative approach, this study explored the perceived impacts of a therapeutic yoga intervention for individuals with Parkinson's disease (PD), and examined the beneficial components of the intervention. Eighteen individuals with PD who completed an eight-week yoga intervention participated in focus groups to determine the general effects, transference of effects, and psychosocial impact of the intervention. Additionally, programmatic aspects of the intervention were explored. A conventional content analysis was used to analyze the data. Themes included expanding PD-related social support, using yoga as a tool to combat PD, increasing body awareness and acceptance of PD, and improved participation in leisure activities. Programmatic components discussed included: components of yoga, dosing, and creating social support. Implications for practitioners include recognizing and respecting limits, using a combination of static and dynamic poses, skillful use of props, and purposely creating opportunities to promote social support.
\end{abstract}

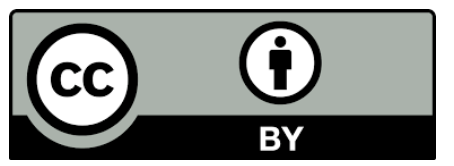

(C) 2020 by the author. This is an open access article distributed under the conditions of the Creative Commons by Attribution License, which permits unrestricted use, distribution, and reproduction in any medium or format, provided the original work is correctly cited. 


\section{Keywords}

Parkinson's disease; therapeutic yoga; social support; complementary and integrative therapy

\section{Introduction}

Following Alzheimer's Disease, Parkinson's disease (PD) is the second most common neurodegenerative disease of adulthood [1]. PD impacts approximately 930,000 individuals in North America in 2020, and is expected to increase to $1,238,000$ by 2030 [2]. PD is a chronic disease that often impacts motor and non-motor skills [3]. Motor skill impairment, including muscle rigidity, bradykinesia, balance, gait, and mobility challenges are most noted with PD [3]. Non-motor impairment in individuals with PD has also been identified as being of concern, and typically includes: fatigue, cognitive changes, impaired quality of life, pain, sleep difficulties, and mood [4].

Psychosocial difficulties (PSDs) have been demonstrated to be of concern in individuals with neurological disorders, even though the disorders themselves present differently in each individual; as is the case in PD. PSDs have been previously categorized using the International Classification of Functioning, Disability, and Health (ICF) [5], and more recently described for people with PD [6, 7]. While PSDs may include body functions, they also include both activities (defined as engagement in a task) and participation (engagement in a life situation) [5]. Broader concerns of PSD for people with PD have been primarily addressed through quantitative studies. For example, Schiavolin et al.[8] studied 80 individuals with PD, and found that while most PSD were related to motor and non-motor symptoms, additional concerns, such as lacking motivation, reduced engagement in community activities, and relationships with friends were also impacted. These data, while important, are limited in describing how these PSDs are experienced by people with PD.

Yoga is a complementary and integrative health intervention that has been used to address PSDs. For example, Van Puymbroeck and colleagues found that yoga helped improve activities and participation for middle-aged and older adults through the reduction of activity constraints [9]. Other studies have demonstrated that yoga has improved PSDs in individuals with other neurological disorders, such as traumatic brain injury [10], chronic stroke [9, 11], and peripheral diabetic neuropathy [12]. Therefore, the purpose of this qualitative study was to explore the perceived psychosocial impacts and the perceived beneficial commonents of a therapeutic yoga intervention for individuals with PD through a qualitative analysis of data.

\section{Methods}

This phenomenological conventional content analysis is a secondary data analysis of qualitative data from a randomized waitlist-controlled pilot study to evaluate a therapeutic yoga intervention for individuals with PD. The primary study is described in detail elsewhere [13]. Approval for the study was obtained from an ethics review board prior to recruitment (\#PRO00041068).

\subsection{Recruitment}

Participants were recruited by physician referral, and must have met the following inclusion/exclusion criteria: 18 years or older, diagnosed with PD, endorse fear of falling, able to 
walk 10 meters (with or without an assistive device), able to speak English, commit to attending all yoga sessions, score between 1.5-3.0 on the Hoehn and Yahr scale of PD progression,[14] score four or higher out of six on the six-item screener for cognitive functioning [15]. Eligible participants who consented to participate in the study, and in focus groups following the study were enrolled and randomly assigned to the treatment group or the waitlist control group. Randomization was done using random number generation, and there were no significant differences in demographics, health status, Hoehn and Yahr scores, or cognitive functioning scores (as measured by the Montreal Cognitive Assessment[16]) between the two groups. All participants engaged in the yoga intervention eventually. After the yoga intervention, all participants (from both the treatment group and waitlist control (WLC) group) were invited to participate in a focus group.

\subsection{Intervention}

The therapeutic yoga intervention was designed and delivered by a Certified Yoga Therapist (CIAYT). A C-IAYT is certified through the International Association of Yoga Therapy. They have been through rigorous training in health conditions, assessment, and using yoga to achieve therapeutic outcomes, and must have met minimum hours of supervised clinical experience. in a face-to-face group format at a university facility twice a week for eight weeks. The yoga intervention was 60 minutes long, and there were 13-14 participants in each class. The yoga sequences included a combination of standing, sitting, and supine positions (see primary article for full table of postures) [13]. Postures, breathing, and meditation were included in all sessions. Individualized modifications of postures were offered to participants throughout the program in order to accommodate individual physical abilities and to ensure participants received the greatest possible benefit. For example, participants who experienced knee pain when they were on the floor were offered alternative postures from a chair so they could experience the benefits of the pose without knee pain. Trained assistants were present for each yoga session, and provided assistance for safety and stability as needed. Participants had the opportunity to talk informally before and after yoga.

\subsection{Data Collection}

Focus groups were conducted following the eight-week yoga intervention for both the experimental group and the waitlist control group. The focus group was semi-structured: the facilitator asked all questions, but participants could make comments and follow-up questions were allowed. Focus groups and were related to general intervention effects, transference of effects, psychosocial impacts, and programmatic aspects (see Table 1 for complete list of focus group questions). Focus group length was an average of 45.96 ranging from 30-55.5 minutes. 
Table 1 Focus Group Questions.

\begin{tabular}{ll}
\hline Content Area & Questions \\
& Tell me about your expectations of this program? \\
Tell me about how this program has impacted you & Now that the program is complete, what benefits did you \\
General Effects & Weceived from the program? \\
& How has the program impacted you in your home? \\
Transference of Effects & How has the program impacted you out in your community? \\
& Will you continue with yoga after the study? \\
& What is it like to be in yoga with other people with Parkinson's? \\
Have you developed new friendships or felt support from & members in this group? If so, please describe. \\
Psychosocial Impacts & Do you feel the program impacted your overall quality of life? \\
& Has the program change your perception of yourself or your \\
& Parkinson's condition? \\
Do you think this type of yoga is appropriate for people with \\
Parkinson's? Why or why not? \\
What parts of the program/sequences/poses did you benefit \\
from most? Why? \\
What parts of the program/sequences poses did you dislike or \\
not benefit from? Why? \\
What changes would you suggest to the program? \\
When you missed a session, what prevented you from coming?
\end{tabular}

\subsection{Data Analysis}

Audio recordings of focus groups were transcribed verbatim and participants were each assigned a pseudonym. The transcripts were analyzed using QSR International's NVivo software. Conventional content analysis was used to analyze the focus group transcripts to understand the perceived impact of the intervention [17]. Additionally, researchers examined programmatic components related to the intervention. Two researchers independently analyzed the data and compared themes. A coding comparison query in NVivo revealed an interrater agreement rate of 98.94\%.

\section{Results}

A total of 27 people (17 males and 10 females) completed the intervention, and out of those 18 completed the focus groups (see Table 2 for demographics of the sample). In total, four focus groups were conducted; one focus group was conducted with participants from the WLC group $(n=5)$ after they completed the yoga intervention, and three focus groups were completed with participants 
from the experimental group $(n=5, n=6, n=2)$. All participants were Caucasian and had an average age of $66.55 \pm 6.36$ years (see Table 3 for additional demographic information for both the total RCT participants and the focus group participants). Four themes emerged related to perceived impact of the intervention: 1) Expanding PD-related social support; 2) Yoga as a tool to combat PD; 3) Increasing body awareness and acceptance of PD; and 4) Improved participation in leisure activities.

Table 2 Sample Demographic Information.

\begin{tabular}{|c|c|c|c|c|}
\hline & \multicolumn{2}{|c|}{ Focus Group Sample } & \multicolumn{2}{|c|}{ Randomized Control Trial Sample } \\
\hline & Percentage & Number & Percentage & Number \\
\hline \multicolumn{5}{|l|}{ Gender } \\
\hline Male & 72.22 & 13 & 62.96 & 17 \\
\hline Female & 27.78 & 5 & 37.04 & 10 \\
\hline \multicolumn{5}{|l|}{ Marital Status } \\
\hline Married & 88.88 & 16 & 88.89 & 24 \\
\hline Widowed & 5.56 & 1 & 3.70 & 1 \\
\hline $\begin{array}{l}\text { Divorced or } \\
\text { separated }\end{array}$ & 5.56 & 1 & 7.41 & 2 \\
\hline \multicolumn{5}{|l|}{ Race } \\
\hline White & 100 & 18 & 100 & 27 \\
\hline \multicolumn{5}{|l|}{ Education } \\
\hline $\begin{array}{l}\text { High school or } \\
\text { less }\end{array}$ & 5.56 & 1 & 7.41 & 2 \\
\hline College & 66.67 & 12 & 59.26 & 16 \\
\hline Post-graduate & 27.77 & 5 & 34.44 & 9 \\
\hline
\end{tabular}

Table 3 Focus Group Demographics.

\begin{tabular}{|c|c|c|c|c|}
\hline & Focus Group A & Focus Group B & Focus Group C & Focus Group D \\
\hline Total & $n=6$ & $\mathrm{n}=5$ & $n=5$ & $n=2$ \\
\hline Group Type & Experimental & Experimental & $\begin{array}{l}\text { Waitlist Control } \\
\text { Group }\end{array}$ & Experimental \\
\hline Age & $\begin{array}{l}65.2 \pm 4.79 \\
(\text { Range }=59-73)\end{array}$ & $\begin{array}{l}61.6 \pm 5.68 \\
(\text { Range }=56-70)\end{array}$ & $\begin{array}{l}71.8 \pm 6.06 \\
(\text { Range }=67-81)\end{array}$ & $\begin{array}{l}70 \pm 1.41 \\
(\text { Range }=69-71)\end{array}$ \\
\hline Gender & $\begin{array}{l}\text { Male: } n=6 \\
\text { Female: } n=0\end{array}$ & $\begin{array}{l}\text { Male: } n=2 \text { Female: } \\
(n=3)\end{array}$ & $\begin{array}{l}\text { Male: }(n=4) \\
\text { Female: }(n=1)\end{array}$ & $\begin{array}{l}\text { Male: }(n=0) \\
\text { Female: }(n=2)\end{array}$ \\
\hline Hoeh \& Yahr Score & $\begin{array}{l}2.08 \pm 0.49 \\
(\text { Range }=1.5-3)\end{array}$ & $\begin{array}{l}2 \pm 0 \\
\text { (Range = 2-2) }\end{array}$ & $\begin{array}{l}2.2 \pm 0.45 \\
\text { (Range }=2-3)\end{array}$ & $\begin{array}{l}3 \pm 0 \\
(\text { Range }=3-3,)\end{array}$ \\
\hline $\begin{array}{l}\text { Montreal Cognitive } \\
\text { Assessment Score }\end{array}$ & $\begin{array}{l}26.5 \pm 1.76 \\
\text { (Range }=24-29)\end{array}$ & $\begin{array}{l}25.4 \pm 1.82 \\
\text { (Range }=23-28)\end{array}$ & $\begin{array}{l}24 \pm 4.30, \text { (Range } \\
=18-29 \text { ) }\end{array}$ & $\begin{array}{l}16 \pm 11.31, \text { (Range } \\
=8-24)\end{array}$ \\
\hline
\end{tabular}




\subsection{Perceived Impacts}

\subsubsection{Expanding PD-related social support}

While participants had been brought together to engage in the yoga intervention, participants found that being with other individuals with PD was particularly meaningful and educational. Charles described the beneficial experience of bonding with the other men in the group, saying "For us fellows, to be here [in yoga] like a little group of guys, we're fighting the same war" [Male, 59 years, experimental participant]. Participants described gaining knowledge of ways to manage PD symptoms from others in the group. Several participants described how they gained insight into various treatment options from their group that they did not get from their treatment provider. Allen described how being part of a group helped him be able to find additional information about treatments and ways to combat PD, saying:

You know I find what happens too when you get together, and you start to network with certain people. Now I'm a veteran, and I spoke to [peers name] about a couple of things with the veterans. And I got information I never would have gotten if I didn't speak to [him]. Again, you meet with Parkinson's people, you share, and you pick up all kinds of useful information. And this yoga class really helped with that [Male, 64 years, experimental participant].

Another participant, Edwin, further expanded on the value of social support by discussing how social connections help him learn about additional complementary and integrative therapies for PD. He stated:

Well, because we're all kind of equal, and we're here for the same reason. Quite frankly, as long as I've had Parkinson's, I still don't know what it is. Any time I meet somebody, I try to find out more information. I mean it's really something that-it can be a whole lot of different things [Male, 73 years, experimental participant].

Additionally, participants discussed that is could be beneficial to formally include more time for socialization during the groups.

\subsubsection{Yoga as a Tool to Combat PD}

Participants described PD as something they were trying to combat. This idea was illustrated in the following exchange between two participants during a focus group:

Allan: I don't want to take this thing lying down. I want to have some outlet in which I can push myself to feel like the Parkinson's and [I] take on a personal relationship. It's good to have some outlet to help you feel like you're fighting back, you're not just a victim [Male, 64 years, experimental participant].

Brad: When I'm doing my walking every morning, and I know- the walking and hydration and exercising, I consider every step forward I take, I envision myself inside of a boxing ring. There's Parkinson's and me. And every time I take a step, I've thrown a punch at Parkinson's, and I don't let it get me. You guys are dead right on that [Male, 64 years, experimental participant].

Allan: We're gonna fight this thing (PD), and this (yoga) is another weapon in the toolbox to fight it with.

The analogy of combat surfaced in other focus groups. Another participant, described PD, saying, "I look at Parkinson's like an individual. You're in a fight with it. You got to aggressively go after it every day through exercise" [Male, 68 years, WLC participant]. Participants also identified specific 
symptoms that improved with yoga, including gait, posture, and balance. For example, one participant discussed their improved gait saying "People have said they really notice a huge difference in me - in my stability, in walking - from when this class started [Female, 68 years, experimental participant]." When asked about perceived improvement since starting yoga another participant discussed posture saying "People have commented to me in a restaurant, 'Boy, your posture. You're sitting up nice and straight'" [Male, 68 years, experimental participant].

\subsubsection{Increasing Body Awareness and Acceptance of PD}

Through the yoga intervention, participants reported they gained awareness of their body, which included strategies to prevent falls and injuries, but also included an awareness of limitations.

Pat discussed how the awareness of specific body movements and postures impacted his fall risk, for example:

I think some of the things related to how you position your feet. Before, if I were getting dizzy, I wouldn't have been thinking about-- I would've been trying to grab for something right away, but I think yoga has made me aware of positioning my feet at a wider stance and things like that [Male, 61 years, experimental participant].

Another participant discussed how the body awareness and the awareness of limitations he developed during yoga helped increased his ability to prevent a fall. He said:

A couple of times I've tripped over curbs, so I've fallen a couple of times. But [yoga] has given me an awareness, so I just think about things a little bit different. If I'm in the shower, and I close my eyes, and I pick my head up, I'm not so much worried about falling anymore. Because I just kind of know it's a part of it. It just has increased my awareness. It put me more in touch with my own limitations [Male, 64 years, experimental participant].

Being aware of and accepting limitations was discussed in conjunction with body awareness through the focus groups. Fran explained how failing to acknowledge limitations could lead to risky behavior and potential risk of falls or injury. Participants also reported the importance of being willing to rest and return to an activity later if they reached their limit for physical activity. For many, yoga highlighted their limitations, yet concurrently increased their awareness and acceptance of those limitations. Fran described this saying

[Yoga] was extremely helpful in understanding that I have a balance problem... even helping me more to make sure I don't go too far on one thing or another. Doing the yoga poses forces you to do things towards your limits... But it also shows me that I don't have the strength to do much of that. Some years ago, it would have been easy to try to ignore that, and just push it to see how far I can go. In this case, to get as far as I can [in yoga poses] without falling-you've seen the person assigned to me who had to hold onto me a lot of times. So that also tells me that while I might be able to do some of that with somebody helping me, but I shouldn't probably take the risk on my own beyond some points [Female, 69 years, experimental participant].

\subsubsection{Improved Participation in Leisure Activities}

The experience of yoga contributed to improved function that was relevant to their daily living with PD. Many of the functional improvements gained during yoga transferred into and enabled more satisfying leisure participation. Leisure pursuits ranged from increased physical activity, to personal hobbies, and increased participation in social functions. One participant who was an 
amateur photographer described how the PD tremors had made it difficult to hold the camera still enough to get the shots they wanted. They described how yoga had improved this saying: "It just makes me enjoy my hobby a whole lot more, because now I can calm myself down, and kind of eliminate - not totally eliminate - but lessen the shakes" [Male, 73 years, experimental participant]. Several participants discussed being able to walk, jog, or engage in their physical activity more effectively after yoga. One participant stated he went from not being able to walk well to walking faster than his wife, and he attributed this change to some physical changes that occurred through yoga. Other participants described feeling less embarrassed by PD after the yoga intervention. One woman said, "Before I didn't want to tell anybody I had Parkinson's... I was embarrassed... now, you know what? It doesn't bother me" [Female, 56 years, experimental participant].

Another woman described how her husband encouraged her to join him for social events, but she always refused because she feared she would be an embarrassment due to PD-related speech symptoms. She stated:

I didn't feel in my head and in my heart that I had the confidence to be out there, because I felt people would notice...me to not be able to carry on a conversation, you know, to stammer and stagger and maybe have my hand get shaking when I'm trying to pour a glass of wine or something like that. But it [yoga]...it's given me the confidence to engage in more social activities [Female, 70 years, experimental participant].

\subsection{Programmatic Elements}

Three subthemes emerged related to programmatic elements; the components of yoga, the social support, and dosing considerations. Each are described below.

\subsubsection{Components of Yoga}

Participants identified specific postures, relaxation, and reciting mantras as helpful aspects of the yoga practice. The most commonly cited poses that participants identified as beneficial were dynamic postures such as lateral bends, spinal twists, and dynamic balancing postures, such as stepping in and out of warrior pose. These were postures that participants stated were helpful to improve coordination and maintain mobility. Additionally, some participants reported that relaxation was beneficial to be able to reduce fall risk because they learned to relax and control their muscles rather than stiffening. One participant described this saying:

I think the other thing that it did for me was relaxing...I think that they spend a fair amount of time with you...doing things that can help you... relax...rather than tightening your muscles. Which in my case would be, I'd want to tighten the muscles so I don't fall. But if you can do it and relax, it also helps you gain strength and balancing ability [Male, 59 years, experimental group].

Participants also highlighted the importance of one-on-one time with the instructor to be sure their technique was correct and prevent injury. One participant described how one-on-one instruction was integral in preventing injury saying,

"For me, I always push myself in trying to do a little bit more than I should do. In the first few lessons I was kind of hurting myself... thanks to [name of yoga therapist] my technique got better. I think working on technique with everybody is really important. Because if you don't have the right technique, you cause pain" [Male, 64 years, experimental participant]. 
Improving technique included the expert use of props to modify poses and increase safety. Props included offering chairs for people who were not comfortable sitting on the floor, and props to help stabilize participants during standing balance (such as back of chairs, walls, or podiums).

In addition to the postures, participants also identified the meditations and mantras they repeated as being motivating and helpful to accepting PD.

\subsubsection{Dosing}

Finally, participants discussed several aspects related to dosing. In each focus group, participants agreed that (60 minutes) was the best length of time, that was not too long to endure, but made it worth traveling to. Several participants indicated they wished it had been more frequent up to three times per week, while others felt that would have been too much traveling. One suggestion was to make the sequence or a video of the sequence available as participants progressed so they could practice outside of group.

\subsubsection{Social Support}

Social support was also discussed in the programmatic context. First, participants mention it was integral to have cheerful and helpful staff and instructor, and that the positivity of the staff motivated them to keep attending. One participant shared how the accountability of seeing peers was a motivating factor:

I think exercise period is good. But when we do it at home independently, it's very easy to go, "I'll do that in an hour [chuckles]," or, "I'll do that tomorrow." But if you're in a-- I think a class is very important because you commit yourself to go to that class. I haven't missed a session, and that's been very important [Male, 81 years, WLC participant].

Participants shared that they made time after class to talk with each other and socialize, but they also recommended ways of instituting more formal social support. One participant suggested "You could ask some of the newer students to talk to the older students that have already been through the class" [Male, 63 years, experimental participant].

Additionally, participants recommended adding a more formal time where people could talk before or after yoga class. One participant suggested that at the beginning participants have the opportunity to share what symptoms yoga has been helping with, and to share the specific pose was working well [Male, 68 years, experimental participant]. Participants in another focus group discussed the possibility of building in social time after the class saying: "What about having a time after the yoga, where people congregate and chat, maybe try some food" [Male, 67 years, WLC participant].

\section{Discussion}

Increased social support is a common outcome experienced by participants in yoga studies [1820]. Van Puymbroeck et al. [20] found that breast cancer survivors identified that being part of a community of people who had experienced similar health challenges was an integral part of the benefits of participating in a therapeutic yoga intervention. In addition to feeling understood, participants valued finding out how others were managing symptoms. Treatment for Parkinson's disease continues to evolve and expand to include more complementary therapies [21]. For 
example, the body research on dance therapy, and Tai Chi as a treatment for Parkinson's disease is growing. Some studies indicate the potential for these in fostering a sense of social support, though a meta-analysis on mind-bodies therapies indicates there is not currently enough research to demonstrate this is higher than treatment as usual [22].

Participants discussed that learning yoga was another helpful tool to be able to combat PD. Results of the present analysis corroborated with the primary findings of this study (see Van Puymbroeck et al. [13]). The primary study reported significant improvements in motor function, gait, and balance before and after participation in yoga, and qualitative results indicate that participants also perceived these improvements [13]. Past research found that participants reported notable improvements in posture, gait, strength, and found yoga reduced bradykinesia, rigidity, and tremors [23]. This may be attributed to the yoga movements themselves, or it may be a combination of improved function and increased body-awareness.

Erden and Guster [24] studied exercise and fall risk among older adults, and found that body awareness (measured by the Body Awareness Questionnaire) was negatively correlated with fall risk in an exercise group. Researchers of another study found correlational evidence linking increased body awareness with better balance [25]. The present study supported the findings of reduced PD-stigma; participants reported increased confidence and decreased embarrassment about their PD symptoms. Mehling et al.'s [26] study of body awareness among mind-body practitioners described qualitatively what body awareness meant saying: "For them, body awareness is an inseparable aspect of embodied self-awareness realized in action and interaction with the environment and world" (p.1). Ghielen et al. [27] conducted a randomized control trial and reported that participants' experienced reduced PD-related stigma after a body awareness intervention that incorporated acceptance and commitment. The experience of participants in the present study seemed to reflect this notion. While some used yoga to slow the progress of the disease, yoga was also used as a way to accept the changes in body functioning brought about by PD. Body awareness was reported as awareness of interaction with their environment (standing in the shower, standing on a rug), and increased body awareness was inseparably connected with the acceptance of the changes that occurred in their body as a result of PD. In the present study, participants attributed better ability to balance to their improved body awareness.

Researchers have demonstrated through multiple studies that yoga improves participation in leisure activities [28-30]. In their study of middle-aged and older adults, Van Puymbroeck, Smith, and Schmid [9] described a potential mechanism for how yoga improves leisure participation. The qualitative findings in the present study supported the proposed mechanism that yoga may modify participants' perception of psychological constraints, leading to increased participation in leisure. Furthermore, the present study adds additional insight into how yoga specifically impacted these psychological constraints. In the initial study, psychological constraints included not having adequate skills, being afraid of getting hurt, and being self-conscious. Participants in the present study described gaining specific skills through yoga that increased their confidence in their ability to prevent a fall or prevent injury during activity. Participants also reported being confident in their ability to recognize their limitations so they would not push beyond their limitations and cause an injury. Additionally, participants in the present study discussed being too self-conscious to engage in social leisure activities prior to the intervention, but reported the social support they received during yoga helped them gain confidence and acceptance of PD. Feeling less inhibition in front of peers who have been through the same illness also occurred in yoga interventions for people with 
breast cancer [31], women going through menopausal transition [19], people with chronic stroke [11], and people with diabetes [32]. This process seems to be the same for people with PD, and this reduced inhibition may have generalized to other settings beyond the yoga class. Thus, the present study supports the proposed mechanism that yoga improves participation in leisure activities by reducing psychological constraints.

\section{Implications for Practitioners}

This study provides insights for yoga therapists and other practitioners who work with this population. First, practitioners should use a combination of static and dynamic poses, and ensure that the practice incorporates relaxation within all the poses. Practitioners should use props to help improved safety, balance, and comfort through the practice. Additionally, practitioners need to provide adequate one-on-one instruction to ensure safety, proper technique and proper use of props. As participants demonstrate their ability to safely engage in poses, practitioners may consider providing resources, such as videos or a handout, for participants to follow at home. To promote social support, practitioners can purposefully imbed opportunities for participants to discuss aspects of PD they are dealing with and what is helping them. Finally, practitioners may consider adding aspects of acceptance and commitment to the practice. Because two of the themes are seemingly contradictory (fighting PD and accepting PD) this dialect might be something that is openly discussed with the group and practiced by pushing limits while accepting limitations.

\section{Limitations and Future Research}

There were limitations to the present study. First, there was a lack of racial diversity, and the applicability of these findings to non-white people with Parkinson's disease is uncertain. Next, which included the possibility that participants who self-selected to participate in the study, were more active and engaged in combating Parkinson's disease than those who declined to participate. Additionally, of those who were in the waitlist control group, only five of 12 completed the focus group. Because this was a secondary analysis with de-identified data, member checking was not possible.

The findings of this study highlight several opportunities for future research. The connection between body awareness and acceptance of PD was not something that was asked about, but a connection that emerged in the data. Future research could more thoroughly examine potential connections between body awareness, PD-related stigma, and acceptance of PD either through more targeted questions, or by a more in-depth quantitative assessment of proprioceptive awareness, interoceptive awareness, and PD-related stigma. Additionally, future research might explore differences in different dosing to determine the most beneficial frequency and duration of practice. Yoga could be compared to other commonly recommended integrative movement-based therapies such as Tai Chi, Dance, and Rocksteady Boxing, to determine if there is a difference in efficacy between the interventions. Finally, future studies should consider controlling for at-home practice. 


\section{Conclusions}

Overall, participants in the therapeutic yoga intervention experienced beneficial psychosocial impacts that extended past the initially targeted outcome of intervention (i.e., improve balance and fear of falling). Yoga contributed to improved function that influenced participants' daily experiences with PD. The group provided participants social support and additional tools to combat the progression of PD, while increasing body awareness and acceptance of limitations. Practitioners who work with this population may consider implementing or referring patients to a therapeutic yoga program to improve psychosocial outcomes, in addition to functional skill improvement.

\section{Acknowledgments}

We acknowledge the assistance of Dr. Kathleen Woschkolup, Dr. Enrique Urrea-Mendoza, Dr. Fredy Revilla, Dr. Kevin Kopera, Leslie Wise, DPT, Nikki Stafford, MBA, OTR, and Stephanie Tanner, MS, for their assistance in the execution of this trial within the Greenville Health System. Additionally, yoga therapist, Peggy Ambler, C-IAYT, who led the yoga intervention, Alysha Walter served as the project manager, the following students assisted in data collection: Spensir Mowrey, Jaesung Park, and Katie Mitchell.

\section{Author Contributions}

Em V. Adams assisted in data analysis, and wrote the methods, results, and discussion sections of the manuscript. Marieke Van Puymbroeck developed the study design, secured funding, served as the PI through the intervention and data collection, and wrote the introduction and edited the manuscript. Brent L. Hawkins assisted in data collection and data analysis. Arlene A. Schmid assisted in developing the study design and edited the manuscript.

\section{Funding}

This study was funded by the College of Health, Education, and Human Development at Clemson University.

\section{Competing Interests}

The authors have declared that no competing interests exist.

\section{References}

1. De Lau LM, Breteler MM. Epidemiology of Parkinson's disease. Lancet Neurol. 2006; 5: 525-535.

2. Marras C, Beck JC, Bower JH, Roberts E, Ritz B, Ross GW, et al. Prevalence of Parkinson's disease across North America. NPJ Park Dis. 2018; 4: 21.

3. Müller B, Assmus J, Herlofson K, Larsen JP, Tysnes OB. Importance of motor vs. non-motor symptoms for health-related quality of life in early Parkinson's disease. Parkinsonism Relat Disord. 2013; 19: 1027-1032.

4. Elbers RG, van Wegen EE, Verhoef J, Kwakkel G. Impact of fatigue on health-related quality of life in patients with Parkinson's disease: A prospective study. Clin Rehabil. 2014; 28: 300-311. 
5. World Health Organization. International classification of functioning, disability, and health. Geneva: WHO; 2001.

6. Vojciechowski AS, Zotz TG, Loureiro AP, Israel VL. The international classification of functioning, disability and health as applied to Parkinson's disease: A literature review. Adv Park Dis. 2016; 5: 29-40.

7. van Uem JM, Marinus J, Canning C, van Lummel R, Dodel R, Liepelt-Scarfone I, et al. Healthrelated quality of life in patients with Parkinson's disease-a systematic review based on the ICF model. Neurosci Biobehav Rev. 2016; 61: 26-34.

8. Schiavolin S, Raggi A, Quintas R, Cerniauskaite M, Giovannetti AM, Covelli V, et al. Psychosocial difficulties in patients with Parkinson's disease. Int J Rehabil Res. 2017; 40: 112-118.

9. Van Puymbroeck $M$, Smith $R$, Schmid A. Yoga as a means to negotiate physical activity constraints in middle-aged and older adults. Int J Disabil Hum Dev. 2011; 10: 117-121.

10. Pering $\mathrm{CH}$, Van Puymbroeck $\mathrm{M}$, Crowe BM, Schmid AA. The influence of yoga on people with traumatic brain injury related to sleep and mood. Open Access J Complement Altern Med. 2020; 1: 67-75.

11. Van Puymbroeck M, Allsop J, Miller KK, Schmid A. ICF- based improvements in body structures and function, and activity and participation in chronic stroke following a yoga-based intervention. Am J Recreat Ther. 2014; 13: 23-33.

12. Van Puymbroeck M, Atler K, Portz JD, Schmid AA. Multidimensional improvements in health following Hatha Yoga for individuals with diabetic peripheral neuropathy. Int J Yoga Therap. 2018; 28: 71-78.

13. Van Puymbroeck M, Walter AA, Hawkins BL, Sharp JL, Woschkolup K, Urrea-Mendoza E, et al. Functional improvements in Parkinson's disease following a randomized trial of yoga. Evid Based Complement Alternat Med. 2018; 2018: 8516351.

14. Goetz CG, Tilley BC, Shaftman SR, Stebbins GT, Fahn S, Martinez-Martin P, et al. Movement disorder society-sponsored revision of the unified Parkinson's disease rating scale (MDSUPDRS): Scale presentation and clinimetric testing results. Mov Disord. 2008; 23: 2129-2170.

15. Callahan CM, Unverzagt FW, Hui SL, Perkins AJ, Hendrie HC. Six-item screener to identify cognitive impairment among potential subjects for clinical research. Med Care. 2002; 40: 771781.

16. Nasreddine Z. Montreal Cognitive Assessment. Québec: Montreal Cognitive Assessment; 2017. Available from: www.mocatest.org.

17. Hsieh HF, Shannon SE. Three approaches to qualitative content analysis. Qual Health Res. 2005; 15: 1277-1288.

18. Conboy LA, Wilson A, Braun T. Moving beyond health to flourishing: The effects of yoga teacher training. ScientificWorldJournal. 2010; 10: 788-795.

19. Crowe BM, Van Puymbroeck M, Linder SM, Francis A, Watt PJ, Francis A. The effects of yoga participation on women's quality of life and symptom management during the menopausal transition: A pilot study. Health Care Women Int. 2015; 36: 1124-1142.

20. Van Puymbroeck, M. Schmid A, Shinew K, Hsieh PC. Influence of hatha yoga on physical activity constraints, physical fitness, and body image of breast cancer survivors: A pilot study. Int J Yoga Therap. 2011; 21: 49-60.

21. Ferry $P$, Johnson $M$, Wallis $P$. Use of complementary therapies and non-prescribed medication in patients with Parkinson's disease. Postgrad Med J. 2002; 78: 612-614. 
22. Kwok YY, Choi KC, Chan LY. Effects of mind-body exercises on the physiological and psychosocial well-being of individuals with Parkinson's disease: A systematic review and meta-analysis. Complement Ther Med. 2016; 29: 121-131.

23. Colgrove $Y$, Sharma N, Kludlng $P$, Potter D. Effect of yoga on motor function in people with Parkinson's Disease: A randomized, controlled pilot sutdy. J Yoga Phys Ther. 2012; 2: 2157-7595.

24. Erden A, Güner SG. Impact of exercise on quality of life, body awareness, kinesiophobia and the risk of falling among young older adults. Cukurova Med J. 2018; 43: 941-950.

25. Gyllensten AL, Hui-Chan WY, Tsang WN. Stability limits, single-leg jump, and body awareness in older Tai Chi practitioners. Arch Phys Med Rehabil. 2010; 91: 215-220.

26. Mehling WE, Wrubel J, Daubenmier JJ, Price CJ, Kerr CE, Silow T, et al. Body Awareness: A Phenomenological Inquiry into the Common Ground of Mind-Body Therapies. Philos Ethics Humanit Med. 2011; 6: 6.

27. Ghielen I, van Wegen EE, Rutten S, de Goede JT, Houniet-de Gier M, Collette EH, et al. Body awareness training in the treatment of wearing-off related anxiety in patients with Parkinson's disease: Results from a pilot randomized controlled trial. J Psychosom Res. 2017; 103: 1-8.

28. Van Puymbroeck M, Payne LL, Hsieh PC. A phase i feasibility study of Yoga on the physical health and coping of informal caregivers. Evidence Based Complement Altern Med. 2007; 4: 519-529.

29. Van Puymbroeck M, Schmid A, Walter A, Hawkins B. Improving leisure constraints in older adults with a rear of falling through Hatha yoga: An acceptability and feasibility study. Int J Gerontol Geriatr Res. 2017; 1: 1-13.

30. Adams E V., Crowe BM, Van Puymbroeck M, Alison CK, Schmind AA. Yoga as a community-based recreational therapy intervention for older adults: A pilot study. Ther Recreat J. 2019; 53: 368380.

31. Van Puymbroeck M, Schmid A, Shinew K, Hsieh PC. Influence of Hatha Yoga on physical activity constraints, physical fitness, and body image of breast cancer survivors: A pilot study. Int J Yoga Therap. 2011; 21: 49-60.

32. Van Puymbroeck M, Atler K, Portz JD, Schmid AA. Multidimensional improvements in health following Hatha Yoga for individuals with diabetic peripheral neuropathy. Int J Yoga Therap. 2018; 28: 71-78.

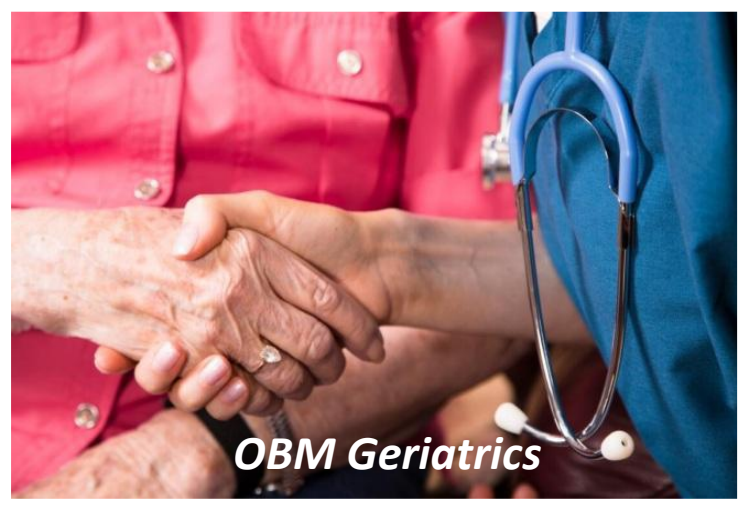

Enjoy $O B M$ Geriatrics by:

1. Submitting a manuscript

2. Joining in volunteer reviewer bank

3. Joining Editorial Board

4. Guest editing a special issue

For more details, please visit: http://www.lidsen.com/journals/geriatrics 